\title{
Introduction to the Special Issue on the IEEE 2003 Custom Integrated Circuits Conference
}

\section{$\mathbf{T}$} HIS Special Issue of the IEEE Journal OF SOLID-STATE CIRCUITS is a selection of papers published in the 2003 IEEE Custom Integrated Circuits Conference. The papers of this selection reflect a continuing trend toward higher performance, lower cost, and further miniaturization. The 20 full papers are grouped into device modeling and radio-frequency papers, mixed-signal papers, and digital papers. Three brief papers conclude the issue.

The first two papers focus on MOSFET modeling techniques. In the first paper, entitled "SP: An Advanced Surface-PotentialBased Compact MOSFET Model," Gildenblat et al. describe the latest advances in surface-potential modeling techniques. The focus of this work is the relation between device physics and circuit simulation in the framework of a practical modeling environment. In the second paper, entitled "High-Frequency Characterization and Modeling of Distortion Behavior of MOSFETs for RF IC Design," Lee and Cheng's HF distortion characterization work indicates that the "low frequency limit" of a MOSFET is much higher than that of a comparable BJT, a useful observation for dealing with distortion behavior in RF IC design.

The next four papers discuss oscillators and quadrature local oscillator generation for a number of applications. In the first paper, "A Study of Injection Locking and Pulling in Oscillators," Razavi studies how the injection of periodic signals into an oscillator may result in locking and pulling phenomena. In the second paper, "Analysis and Design of Injection Locked LC Dividers for Quadrature Generation," Mazzanti et al. describe analytical expressions for locking range, quadrature accuracy, and phase noise for a class of divider topologies. In the third paper, entitled "A Subharmonically-Injected LC Delay Line Oscillator for 17-GHz Quadrature LO Generation," Ma and Long propose a delay line oscillator for simultaneous frequency multiplication and quadrature signal generation. The fourth paper by Temporiti et al., entitled "A $700-\mathrm{kHz}$ Bandwidth $\Sigma \Delta$ Fractional Synthesizer with Spurs Compensation and Linearization Techniques for WCDMA Applications," proposes a 2.1-GHz frequency synthesizer with $35-\mathrm{Hz}$ resolution obtained thanks to a very large PLL bandwidth.

The next paper, entitled "A 4-91-GHz Traveling-Wave Amplifier in a Standard 0.12- $\mu \mathrm{m}$ SOI CMOS Microprocessor Technology," by Plouchart et al., demonstrates a very highbandwidth design implemented as a seven- and five-stage traveling-wave amplifier. The amplifiers operate at low voltage and exhibit a relatively low noise figure throughout the bandwidth.

The next two papers deal with data conversion. "Radio Frequency Digital-to-Analog Converter," by Luschas et al., describes an RF narrow-band sigma-delta DAC designed to

Digital Object Identifier 10.1109/JSSC.2004.832197 demonstrate a different DAC architecture where the DAC output is controlled by an oscillating waveform. The goal is to mitigate the troublesome switching distortion and clock jitter effects in high-speed DACs. In the next paper, entitled "A 1.8-V 67-mW 10-bit 100-MS/s Pipelined ADC Using Time-Shifted CDS Technique," Li and Moon discuss design of a pipelined ADC using a time-shifted correlated double sampling technique to reduce finite opamp gain error in order to achieve balanced performance for speed, power, and accuracy.

The next two papers focus on imager and display circuit design. In the first paper, "Amorphous Silicon Thin Film Transistor Circuit Integration for Organic LED Displays on Glass and Plastic," by Nathan et al., the authors present a design of TFT drive circuits for active matrix organic light-emitting diode (OLED) displays, where the OLED layer was directly integrated on top of the TFT drive circuits. Next, Acosta-Serafini et al. propose a high dynamic range VGA sensor in the paper entitled "A 13 " VGA Linear Wide Dynamic Range CMOS Image Sensor Implementing a Predictive Multiple Sampling Algorithm With Overlapping Integration Intervals." The sensor's operation is based on a variable integration capacitance at the pixel level in combination with a feedback mechanism to extend saturation intensity levels.

The next paper, entitled "Statistical Leakage Current Reduction in High Leakage Environments using Locality of Block Activation in Time Domain," by Choi et al., describes a new leakage current reduction methodology for realizing statistical leakage current reduction using a time locality of activation probability of a circuit block.

The next eight papers discuss digital design issues, in particular low power, content addressable memory (CAM), video compression, and SOI technology. The first two papers deal with low power reduction techniques in flip-flops and CAMs. "Standby Power Reduction Using Dynamic Voltage Scaling and Canary Flip-Flop Structures," by Calhoun and Chandrakasan, analyzes various flip-flop structures and proposes a closed-loop approach using canary flip-flops. Measurements reveal that this approach improves power by $40 \times$ in a $0.13-\mu \mathrm{m}$ dual- $V_{T}$ test chip, which in turn is $2 \times$ better than the open-loop approach. The next paper describes a novel pipelined scheme to reduce power consumption in "A Low-Power Content Addressable Memory (CAM) Using Pipelined Hierarchical Search Scheme," by Pagiamtzis and Sheikoleslami.

The third and fourth papers address issues related to clock/ signal distribution. "Resonant Clocking Using Distributed Parasitic Capacitance," by Drake et al., suggests ways of accelerating clock trees with local clock regeneration schemes. Drost $e t$ $a l$. report results from wireless chip-to-chip communication experiments in their paper entitled "Proximity Communication."

Next, Hazucha et al. demonstrate a $10 \times$ improved reliability in SER tolerant latches in a test chip containing both standard 
and SER tolerant latches in the paper entitled "Measurements and Analysis of SER Tolerant Latch in 90-nm Dual- $V_{t}$ CMOS Process." Garrett et al. present an architecture for $4 \times 4$ 16QAM MIMO spherical decoder that achieves $38.8 \mathrm{Mb} / \mathrm{s}$ over a $5-\mathrm{MHz}$ channel using only approximately $10 \mathrm{~mm}^{2}$ in a $0.18-\mu \mathrm{m}$ CMOS process in the paper "Silicon Complexity for Maximum Likelihood MIMO Detection Using Spherical Decoding."

The seventh paper, by Farjad-Rad et al., entitled "A 33-mW 8-Gb/s CMOS Clock Multiplier and CDR for Highly Integrated I/Os," describes a compact non-LC low-power low-jitter 8-Gb/s CDR circuit using injection-locking for jitter suppression and phase interpolation in high-bandwidth SOC solutions. A mobile terminal for personal visual communication is gaining popularity and to realize an ultra-low-power and high-quality real-time MPEG4 video codec in the terminal, a highly efficient motion estimation process is essential. The next paper, by Miyama et al., entitled "A Sub-mW MPEG-4 Motion Estimation Processor Core for Mobile Video Application," features a gradient search algorithm that reduces computational complexity to 15 MOPs.

This Special Issue concludes with three brief papers. "Analysis and Modeling of Bang-Bang Clock and Data Recovery Circuits," by Lee et al., proposes a large-signal model for the characterization of jitter in bang-bang phase detectors. Partially depleted SOI (PD SOI) circuits possess inherent BJT leakage affecting the functionality of the circuit. Nanua and Blaauw address the PD SOI noise issues in a paper entitled "Noise
Analysis Methodology for Partially Depleted SOI Circuits" and present a noise model to account for the floating body and the BJT effects. The final paper, by Prokop et al., "An Eight-User UMTS Channel Unit Processor for 3GPP Base Station Applications," describes a multiuser W-CDMA baseband channel unit processor for cellular base-station applications that exceeds 3GPP performance requirements.

We would like to extend our thanks to the authors for their work in submitting and revising their manuscripts. Learning about novel work and advances of the art has been highly gratifying. We also wish to express our deepest gratitude to our reviewers for their efforts and dedication. This Special Issue would not have been possible without their expert advice.

\author{
EDOARDO CHARBON \\ EPFL \\ Lausanne, 1015 Switzerland \\ AlberT Z. H. WANG \\ Integrated Electronics Laboratory \\ Illinois Institute of Technology \\ Chicago, IL 60616 \\ SREEDHAR NATARAJAN \\ MoSys Inc. \\ Stittsville, ON K2S 1W6 Canada
}

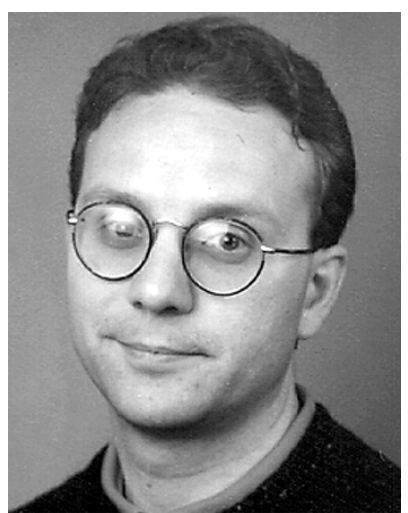

Edoardo Charbon (M'92) received the Diploma in electrical engineering from the Swiss Federal Institute of Technology (ETH), Zürich, Switzerland, in 1988, the M.S. degree in electrical and computer engineering from the University of California at San Diego in 1991, and the Ph.D. degree from the University of California at Berkeley in 1995. His doctoral work focused on performance-directed constraint-based analog and mixed-signal physical design automation and accelerated substrate extraction techniques.

From 1995 to 2000, he was with Cadence Design Systems, where he was the architect of the company's initiative for intellectual property protection. In 2000, he joined Canesta Inc. as its Chief Architect, leading the development of wireless three-dimensional (3-D) CMOS image sensors. Since November 2002, he has been a member of the Faculty of the Swiss Federal Institute of Technology, working in the field of 3-D sensors and ultra-low-power wireless embedded systems. He has consulted for numerous organizations, including Texas Instruments Incorporated, Hewlett-Packard, and the Carlyle Group. He has published over 55 articles in technical journals and conference proceedings and two books, and he holds six patents. His research interests include 3-D micro-imaging, radio-frequency integrated circuits, intellectual property protection, substrate modeling and characterization, superconducting parasitic analysis, and micromachined sensor design.

Dr. Charbon has served as Guest Editor of the IEEE TRANSACTIONS ON COMPUTER-AIDED DESIGN OF INTEGRATED CIRCUITS AND SYSTEMS and as a Member of the Technical Committee of the IEEE Custom Integrated Circuits Conference since 1999. 


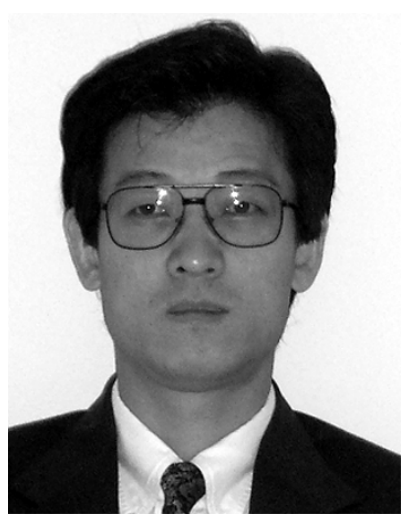

Albert Z. H. Wang (M'95-SM'00) received the B.Eng. and Ph.D. degrees in electrical engineering from Tsinghua University, China, and the State University of New York at Buffalo, in 1985 and 1996, respectively.

He was with National Semiconductor Corporation until 1998, when he joined the Faculty of Electrical and Computer Engineering of the Illinois Institute of Technology, where he is an Associate Professor and directs the Integrated Electronics Laboratory. His research interests center on analog/mixed-signal/RF ICs, advanced on-chip ESD protection, IC CAD and modeling, and SoCs and semiconductor devices. He is the author of the book On-Chip ESD Protection for Integrated Circuits (Kluwer, 2002) and more than 70 papers in the field, and holds several U.S. patents. He is a frequent speaker at various industrial, academic, and international forums, and a frequent consultant to the IC industry.

Dr. Wang is an Editor for the IEEE Electron DEVICE LETTERS, an Associate Editor for the IEEE TRANSACTIONS ON CIRCUITS AND SYSTEMS-PART I, a Guest Editor for the IEEE JOURNAL OF SOLID-STATE CIRCUITS and a Guest Editor-in-Chief for the IEEE TrANSACTIONS ON ELECTRON DEVICES. He was an Associate Editor for the IEEE TRANSACTIONS ON CIRCUITS AND SYSTEMS-PART II. He is an IEEE Distinguished Lecturer for the Electron Devices Society and the Solid-State Circuits Society. He serves as TPC Member, Sub-Committee Chair, and Session Chair for many conferences, including IEEE CICC, RFIC, APC-CAS, ASP-DAC, ISCAS, ICSICT, and NewCAS. He received the CAREER Award from the National Science Foundation in 2002 and the Sigma Xi Award for Excellence in University Research from the Illinois Institute of Technology in 2003.

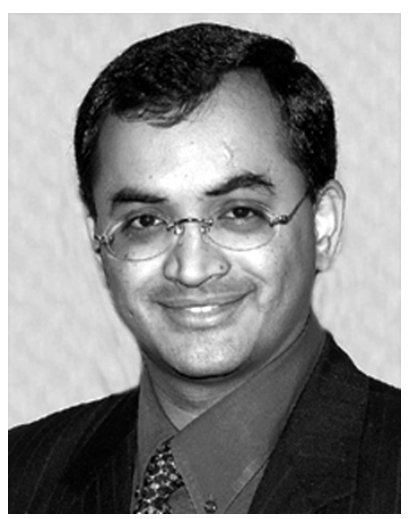

Sreedhar Natarajan (M'97-SM'01) received the M.S. degree in computer engineering from the University of Southwestern Louisiana, Lafayette.

He is currently a Design Manager for embedded memory development at MoSys Incorporated, Ottawa, Canada. He has 15 years experience in memory design, including working for Paradigm Technology, Texas Instruments previously in the areas of SRAM, DRAM, and FRAM design. He currently serves on the technical advisory council for many universities on memory-related projects and is an adjunct member of faculty at the University of North Carolina, Charlotte. He coauthored the book SOI Design: Analog, Memory and Digital Design (Kluwer, 2001). He has mentored various doctoral students at recognized universities on memory-related projects. He is a very strong advocate of emerging memory technologies to the semiconductor memory industry. He has over 15 publications and over 25 filed and issued patents.

Mr. Natarajan serves on various international conference committees including IEEE ISSCC, CICC, ISLPED, SOC, and the VLSI Symposium, and also currently serves on the IEEE Standards board (NesCOM). He has been an invited speaker at various IEEE international conferences and academic institutions. He has hosted many panel discussions and tutorials at major conferences including IEEE ISSCC, CICC, and the VLSI Symposium. He is a Guest Editor for IEEE Journal OF Solid-STATE CIRCUITS for the CICC 2003 and ISSCC 2004 Special Issues. He is the recipient of the IEEE Circuits and Systems Outstanding Service Award in 2001. He is the past chairman for the Dallas Chapter of the IEEE Solid-State Circuits Society. His past IEEE activities include chair of IEEE Solid-State Circuits Society, Dallas Chapter 2000-2001 as a program committee member of the IEEE Dallas Circuits and Systems. He is currently the Vice Chair for IEEE Joint Chapters, Ottawa, Canada. 